\title{
Tu B01
}

\section{Broadband Seismic - Opportunities and Challenges}

M.C. Tanis* (BP America Inc.)

\section{SUMMARY}

Broadband or extended bandwidth seismic over the last few years has grown to become nearly the standard for marine acquisition method while the acquisition and processing of ultra-low and high frequencies still remain a challenge for both marine and land. These seismic broadband solutions range from acquisition based approaches using various purpose built marine streamer configurations to processing based solutions that aim to remove the effects of the ghosts from the conventional seismic data. 
Broadband or extended bandwidth seismic over the last few years has grown to become nearly the standard for marine acquisition method while the acquisition and processing of ultra-low and high frequencies still remain a challenge for both marine and land. These seismic broadband solutions range from acquisition based approaches using various purpose built marine streamer configurations to processing based solutions that aim to remove the effects of the ghosts from the conventional seismic data.

While acquisition based broadband solutions in general provide an effective and reliable solution, for the conventional seismic, however, this matter is more complicated. In recent years a multitude of broadband processing workflows and solutions have been proposed in the industry. While all these various methods and workflows aim to extend the seismic bandwidth on both the low and high end of the spectrum, there are still many questions to answer to reach an industry-wide standard such as

- What benefits do these workflows provide over post-stack noise attenuation and spectral shaping?

- How effective are they compared to various acquisition approaches?

- What are the data assumptions for a successful outcome in regards to $\mathrm{S} / \mathrm{N}$, sampling, tow depths, amplitude, phase etc?

- How different approaches compare relative to each other?

- What issues and challenges these approaches present for 4D seismic?

It is important that the industry converges on a set of guidelines addressing these important questions.

Regardless of its acquisition or processing origin, another important aspect for broadband seismic is, it requires more than ever the input and involvement of the integrated geoscientists for establishing and revising interpretation and analysis guidelines to extract maximum value from the broader band seismic on their workstation. 